Revista Perspectivas Online: Biológicas \& Saúde Junho de 2017, Vol.7, n 24 , p. 40-46

ISSN: 2236-8868 (Online)

DOI: $10.25242 / 886872420171160$

\title{
USO TERAPÊUTICO DA POMADA DE PRÓPOLIS EM DIFERENTES FERIDAS CRÔNICAS
}

\author{
Ana Paula Rodrigues Silva ${ }^{1}$, Ana Paula de Souza Soares ${ }^{1}$, Camila da Silva Pessanha ${ }^{1}$, Chrisson \\ Monteiro Roza', Lavínia Saint'Clair Tavares', Danielle de Menezes Silva, Mayara Monteiro \\ Cardoso, Thaís Aparecida de Castro Palermo ${ }^{2}$, Carolina Magalhães dos Santos ${ }^{2}$ \& Aline Teixeira \\ Marques Figueiredo Silva ${ }^{2 *}$
}

\begin{abstract}
RESUMO
SILVA, A.P.R.; SOARES, A.P.S.; PESSANHA, C.S.; ROZA, C.M.; TAVARES, L.S.; SILVA, D.M.; CARDOSO, M.M.; PALERMO, T.A.C.; SANTOS, C.M. \& SILVA, A.T.M.S. Uso terapêutico da pomada de própolis em diferentes feridas crônicas. Perspectivas Online: Biológicas e Saúde, v.7, n.24, p. 40-46, 2017.

$\mathrm{O}$ uso indiscriminado e prolongado de antimicrobianos sintéticos tem levado à seleção de microrganismos patogênicos mutantes resistentes a esses compostos. A própolis é considerada um dos produtos naturais de maior destaque, conhecida pelas diversas propriedades biológicas que possui e assim utilizada como antimicrobiano, antioxidante, antiinflamatório, imunomodulador, cicatrizante, anestésico, entre outras funções. $\mathrm{O}$ estudo teve como principal objetivo: Avaliar a influência da pomada de própolis em concentração de $30 \%$ na cicatrização de diferentes tipos de úlceras. Foi realizado em uma Unidade de Saúde do município de Campos dos Goytacazes, que atua especificamente com tratamento de

e equipe de pesquisa foram treinados pela equipe de pesquisa para realizar a troca de curativos com a pomada de própolis diariamente, sendo 05 pacientes, avaliados semanalmente através da mensuração da lesão e observação de quantidade de exudato, odor, entre outros fatores envolvidos na avaliação (através de instrumento específico), além do registro fotográfico das lesões para registro e acompanhamento. Os resultados revelaram eficácia da terapêutica alternativa; sendo empregado baixo custo, através de um produto viável; com facilidade na troca da cobertura; e curto tempo médio de cicatrização (45 dias) com $\mathrm{DP}=24,1$ dias, com trocas diárias dos curativos.
\end{abstract} lesões cutâneas, onde os profissionais da Unidade

Palavras-chaves: Ferimentos; Lesões; Cicatrização de feridas; Própolis. 


\begin{abstract}
The indiscriminate and prolonged use of synthetic antimicrobials has led to the selection of mutant pathogenic microorganisms resistant to these compounds. Propolis is considered one of the most outstanding natural products, known for its biological properties and thus used as antimicrobial, antioxidant, anti-inflammatory, immunomodulatory, healing, anesthetic, among other functions. The main objective of this study was to evaluate the influence of propolis ointment on the healing of different types of ulcers. It was carried out in a Health Unit of the municipality of Campos dos Goytacazes, which works specifically with treatment of cutaneous lesions, where the

professionals of the Unit and research team were trained by the research team to perform the exchange of dressings with the propolis ointment daily, With 5 patients being evaluated weekly by measuring the lesion and observing the amount of exudate, odor, among other factors involved in the evaluation (through a specific instrument), as well as the photographic registry of lesions for recording and follow-up. The results revealed efficacy of the alternative therapy; Being used low cost, through a viable product; With easy exchange of coverage; And short healing time (45 days) with $\mathrm{PD}=24.1$ days, with daily dressing changes.
\end{abstract}

Key-words: Wounds; Injuries; Wound healing; Propolis.

\footnotetext{
${ }^{1}$ Acadêmicos de Enfermagem - ISECENSA - - Rua Salvador Correa, 139, Centro, Campos dos Goytacazes, RJ, CEP: 28035-310, Brasil;

${ }^{2}$ Institutos Superiores de Ensino do CENSA - ISECENSA - Laboratório de Estudos em Saúde Pública - Rua Salvador Correa, 139, Centro, Campos dos Goytacazes, RJ, CEP: 28035-310, Brasil.

(*) e-mail: alinemrqs13@gmail.com

Data de chegada: 19/04/2017 Aceito para publicação: 22/05/2017
} 


\section{INTRODUÇÃO}

Nos últimos anos, tem-se verificado um crescente interesse pela medicina popular, tendo em vista a facilidade na manipulação e o baixo custo através da utilização de produtos naturais no controle de algumas enfermidades. Entre estes produtos, a própolis, proveniente das abelhas da espécie Apis mellifera, apresenta grande aceitação devido a suas propriedades terapêuticas (BARBOSA et al., 2009; BEZERRA et al., 2013).

A própolis é considerada atualmente um dos produtos naturais de maior destaque, conhecida pelas diversas propriedades biológicas que possui e assim utilizada como antimicrobiano, antioxidante, antiinflamatório, imunomodulador, hipotensor, cicatrizante, anestésico, entre outras funções. Essas propriedades se encontram relacionadas com sua composição química, que apresenta, até o momento, cerca de 200 elementos já identificados, sendo os principais agrupados em: flavonóides, ácidos graxos, álcoois, aminoácidos, vitaminas e minerais (BARBOSA et al., 2009).

A ação da própolis na regeneração e na granulação dos tecidos é discutida por diversos autores (BARBOSA et al., 2009; BEZERRA et al., 2013; SANTOS et al., 2007; VARGAS et al., 2004). Nesta perspectiva, a própolis é sugerida como produto que favorece a cicatrização, além de sua propriedade antibiótica natural e principalmente desprovida de efeitos colaterais, o que não acontece com os antibióticos sintéticos, apresentando também baixo custo em relação às coberturas utilizadas atualmente, tornando-se acessível à população (BARBOSA et al., 2009; GREGORY et al., 2002).

O uso indiscriminado e prolongado de antimicrobianos sintéticos tem levado à seleção de microrganismos patogênicos mutantes resistentes a esses compostos, tornando o uso de antimicrobianos de origem natural uma alternativa eficaz e econômica. O problema da resistência microbiana é crescente e a perspectiva de uso de drogas antimicrobianas no futuro é incerta. Portanto, devem ser tomadas atitudes que possam reduzir este problema como, por exemplo, controlar o uso de antibióticos, desenvolver pesquisas para melhor compreensão dos mecanismos genéticos de resistência e continuar o estudo de desenvolvimento de novas drogas, tanto sintéticas como naturais (VARGAS et al., 2004).

Estima-se que, em $80 \%$ a $85 \%$ dos casos de úlceras de perna, sejam provocados por insuficiência venosa crônica e que $5 \%$ a $20 \%$ decorram de isquemia por insuficiência arterial, geralmente por progressão de aterosclerose. As afecções clínicas que, com frequência, acompanham o quadro são o diabetes mellitus DM e a hipertensão arterial sistêmica - HAS (OLIVEIRA et al., 2010). O estudo apresentou como objetivos: Acompanhar a evolução do processo cicatricial das úlceras tratadas com própolis nos indivíduos estudados e Avaliar a influência da pomada de própolis em concentração de $30 \%$ na cicatrização de diferentes tipos de úlceras.

\section{METODOLOGIA}

O estudo foi desenvolvido por meio de uma pesquisa experimental, exploratória e descritiva, que envolveu intervenção, análise de resultados e demais observações relativas ao processo de cicatrização das úlceras de diferentes etiologias diante do uso de pomada de própolis na concentração de $30 \%$, conforme apontam estudos anteriores: BEZERRA et al., 2009; SANTOS et al., 2007; VARGAS et al., 2004.

A Amostra de conveniência final foi de 05 indivíduos, sendo utilizados como critérios de inclusão: portadores de feridas crônicas, que realizassem curativos diários, no Centro de Tratamento ao Pé Diabético, de ambos os sexos, que aceitassem participar da pesquisa, através da assinatura do Termo de Consentimento Livre e Esclarecido (TCLE), e que apresentassem diferentes tipos de lesões. Como

Foi realizada capacitação com a equipe da Unidade, no sentido de estabelecer protocolo de troca dos curativos utilizando a pomada de própolis na concentração de $30 \%$ que foi utilizada diariamente em todos os pacientes selecionados para a pesquisa, e o registro, evolução as medidas semanais foram realizados pela

Persp. online: biol. \& saúde, Campos dos Goytacazes, 24 (7), 40-46, 2017

seer.perspectivasonline.com.br 
equipe de pesquisa, identificando e descrevendo a evolução e as mudanças fisiológicas durante o processo de cicatrização das feridas, ou seja, as características relacionadas ao tipo de tecido no leito e na margem da ferida, tipo de exsudato e avaliação da dor e medidas, bem como outras características necessárias ao desenvolvimento do estudo, bem como registro fotográfico das feridas.

Para avaliação do processo cicatricial, foi utilizada a técnica de medição por meio das medidas de comprimento, largura, por meio do Coeficiente de Correlação de Pearson, avaliando-se a relação entre as medidas médias horizontais (MH), verticais (MV), além da análise do tempo de cicatrização e condições gerais da ferida.

A efetividade da terapêutica foi avaliada registrando-se a condição da ferida com a documentação fotográfica, o tipo de tecido, presença de tecido necrótico, sinais de infecção, tipo de exsudato, volume e odor, mensuração das extensões verticais e horizontais, e a duração da ferida. Tais avaliações foram realizadas durante a execução dos curativos diários em ficha de evolução da própria Unidade de Saúde.

O estudo foi submetido ao Comitê de Ética do ISECENSA, e recebeu aprovação sob o número de protocolo CAAE: 57533816.5.0000.5524.

\section{RESULTADOS}

De acordo com o gráfico 01, relacionando-se ao estado geral dos pacientes participantes da pesquisa, ressaltamos que a totalidade $(100 \%)$, realiza tratamento medicamentoso para hipertensão arterial, e $40 \%$ faz uso regular de medicamentos para o tratamento de diabetes mellitus, fatores que podem interferir diretamente na condição de cicatrização das feridas, prolongando o tempo de recuperação do paciente. Em relação ao consumo de álcool e tabagismo, 100\% relatam não fazer uso, fatores que favorecem o processo cicatricial das feridas.

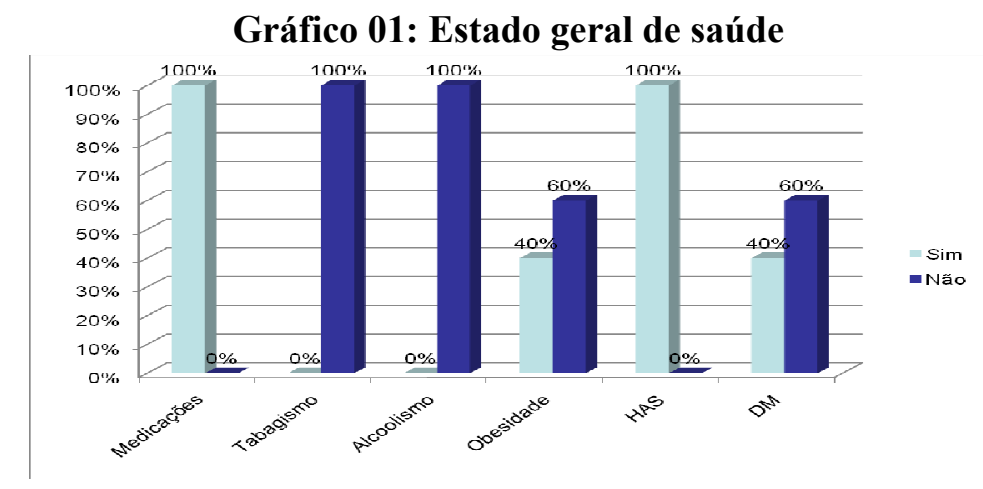

Em comparação aos dados literários encontrados, as informações relacionadas ao uso de tabaco atingiram menor escala nos resultados encontrados, 30\% (SANTOS et al., 2007), assim como a ausência do hábito de ingestão bebida alcoólica nos indivíduos envolvidos na pesquisa. A população estudada apresentou média etária de 55 anos de idade, com desvio padrão 15,5, em concordância com BORGES et al., 2016. A dislipidemia, tabagismo, obesidade e vida sedentária, tornam-se fatores importantes para o aumento de tempo na cicatrização das lesões cutâneas (SANTOS et al., 2007).

Conforme o gráfico 02 , apenas $20 \%$ dos pacientes relatou dor no local da lesão, prévia ao tratamento, relatando melhora do quadro de dor com o uso da pomada de própolis, $40 \%$ apresentou odor forte nos locais das lesões, o que pode nos sugerir grande carga infecciosa, característica das úlceras venosas crônicas, e apenas $20 \%$ da amostra apresentou sangramento intenso durante a troca dos curativos, $60 \%$ das lesões apresentaram quantidade significativa de secreção, em acordo com as descrições clínicas das úlceras venosas crônicas com alta carga infecciosa, tornando-as lesões de difícil manejo, e conseqüente aumento no tempo de cicatrização (SANTOS et al., 2007).

Persp. online: biol. \& saúde, Campos dos Goytacazes, 24 (7), 40-46, 2017 
De acordo com o gráfico $03,40 \%$ das úlceras avaliadas apresentaram ardência no momento da aplicação da pomada de própolis, com melhora do quadro de ardência após alguns minutos da aplicação, o que pode ser explicado pela concentração de álcool necessária para estabilidade do princípio ativo. Dos pacientes avaliados, $40 \%$ faziam uso de atadura elástica de compressão no membro afetado, que facilita o retorno venoso, favorecendo cicatrização, e $60 \%$ não fazia uso de atadura elástica de compressão, o que pode dificultar a cicatrização da úlcera (SANTOS et al., 2007).

\section{Gráfico 02: Sinais e sintomas prévios}

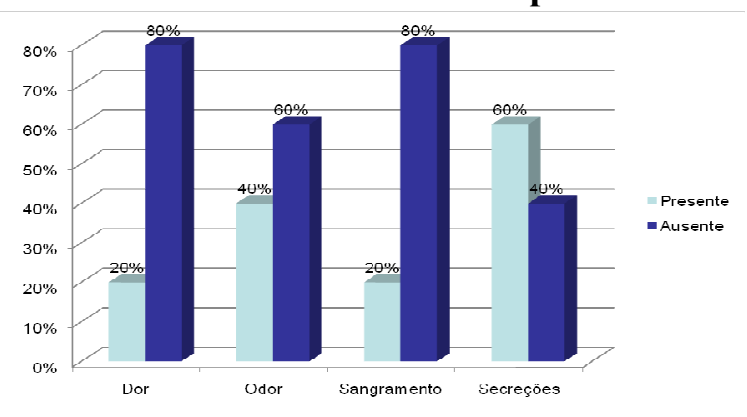

Gráfico 03: Avaliação das úlceras

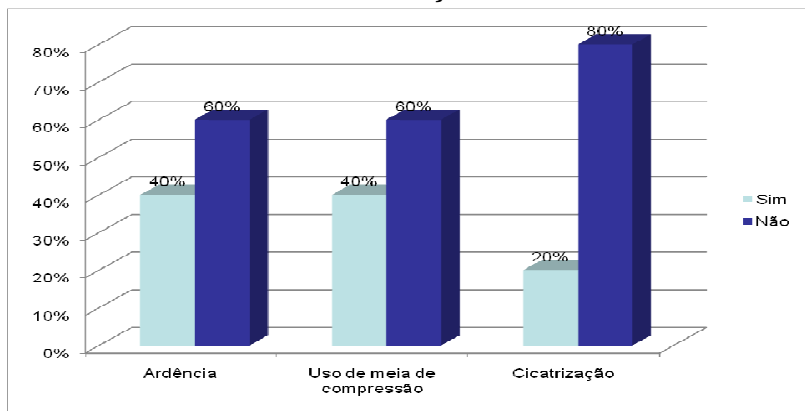

Estudos anteriores apontam a redução de odor em 53,3\% após a introdução da terapêutica com a própolis, bem como a diminuição da sensação dolorosa do paciente após o início do tratamento com a pomada e própolis (BARBOSA et al., 2009).

Em relação à cicatrização das úlceras, apenas $20 \%$ dos pacientes apresentou fechamento completo da ferida (gráfico 03), que pode ser justificado pelo encerramento das atividades da Unidade de Saúde onde a pesquisa foi realizada, interrompendo as atividades da equipe de pesquisa.

Conforme os quadros 01 e 02 (abaixo), as lesões sofreram uma redução em relação à medida horizontal $(8,6 \mathrm{~cm}$ para $5,4 \mathrm{~cm})$ e vertical $(11,8 \mathrm{~cm}$ para $5,4 \mathrm{~cm})$, com tempo médio de cicatrização de 45 dias, apresentando um desvio padrão de 24,1 dias.

\section{Quadros 01 e 02: Avaliação do processo cicatricial}
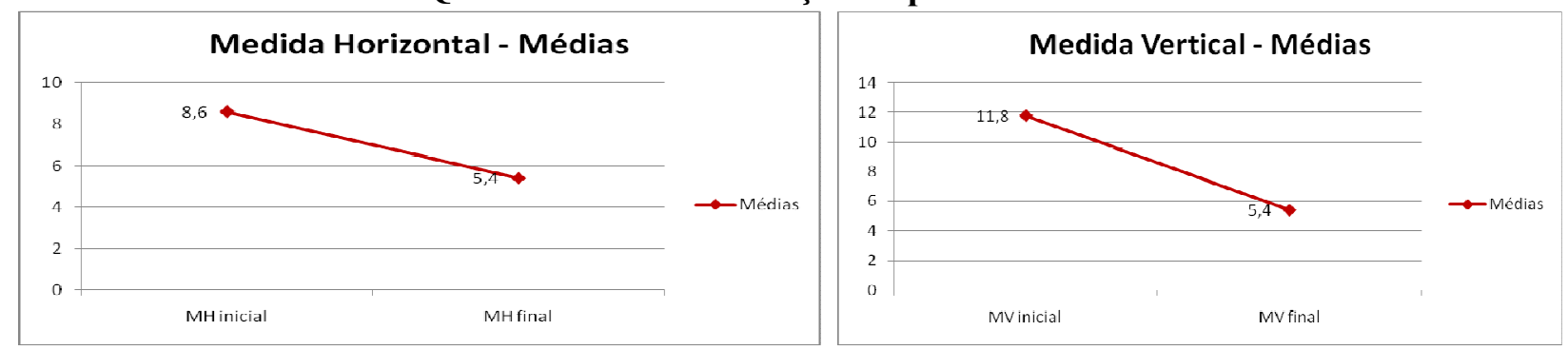

Apontamentos literários realizados a partir de estudos anteriores referem tempo de cicatrização de feridas em uso de pomada de própolis como escolha terapêutica, com média de 65 dias (BARBOSA et al., 2009; SANTOS et al., 2007), o que pode confirmar a eficácia da pomada de própolis como antimicrobiano eficaz na cicatrização das feridas, impactando positivamente na melhora da qualidade de vida dos usuários, sendo uma alternativa de baixo custo, e boa efetividade.

Nas fotos 01 e 02 (abaixo), identificamos o registro fotográfico da evolução de cicatrização das feridas de dois pacientes: foto 01- Paciente A, foto 02- Paciente B, acompanhados pelo projeto. Em ambos os pacientes foi feito uso da pomada de própolis na concentração de $30 \%$ diariamente, com resultado 
positivo para o fechamento da lesão nos dois casos fotografados, o que confirma a eficácia da terapêutica proposta.
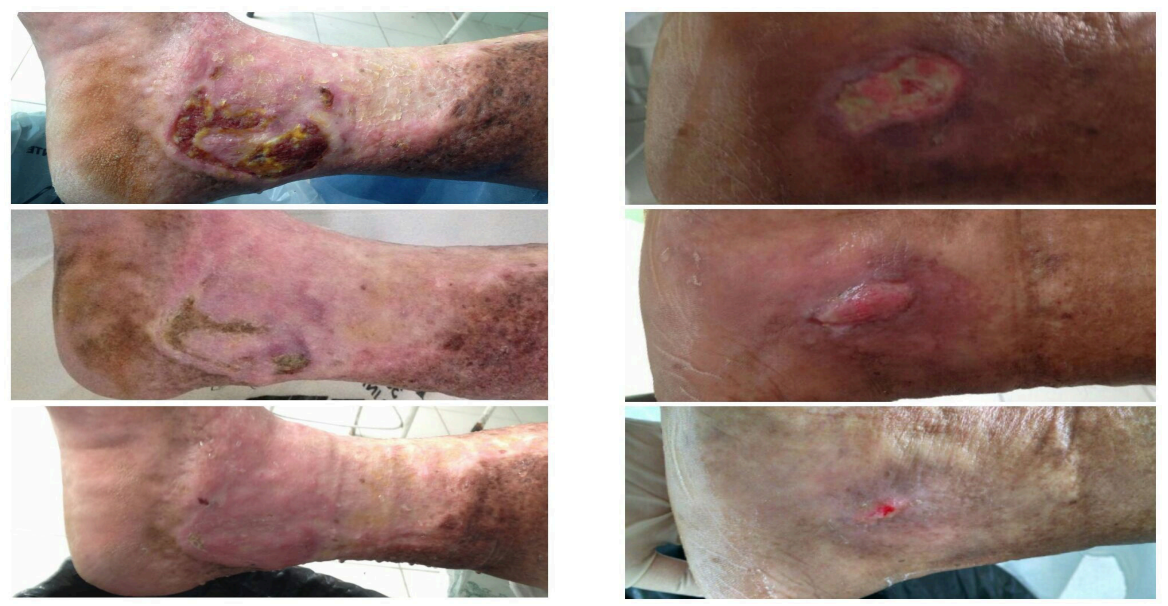

Fotos 01(paciente A) e 02 (paciente B): Evolução das lesões de dois pacientes Acompanhados pela pesquisa após 60 dias de tratamento com a pomada de própolis a $30 \%$.

\section{CONCLUSÃO}

O presente estudo demonstrou experimentalmente os resultados da aplicação de uma cobertura não convencional, à base de própolis na concentração de $30 \%$, em pacientes portadores de feridas crônicas.

A complexidade do processo de cicatrização em pacientes portadores de feridas requer cuidados especiais tanto no que diz respeito aos cuidados diretos com a terapêutica utilizada no curativo, quanto ao controle glicêmico, dos níveis pressóricos, da nutrição e repouso, e demais condições que possam favorecer um ambiente ideal para a epitelização da lesão.

Diversos estudos têm sido realizados tratando a terapia com a própolis em concentrações diferentes, porém, somente a análise dos resultados de um estudo experimental, com uma amostra ampliada, poderá evidenciar maiores esclarecimentos sobre a utilização do produto em diferentes tipos de lesões, bem como dos seus efeitos sobre o processo de cicatrização e de possíveis desconfortos, sensibilidades e reações adversas ao paciente, embora nenhuma complicação ou desconforto tenha sido identificado nos estudos anteriores.

O tratamento e a cura de lesões deve ser o foco em estudos como este, e somente através de métodos de testagem da ação da própolis, ou outros métodos alternativos poderemos comprovar a eficácia da ação da própolis, reafirmando o significante papel da enfermagem no desenvolvimento de novas alternativas para o tratamento de feridas, bem como contribuindo para o fortalecimento da autoestima de pacientes portadores desse tipo de lesão.

Desta forma, o estudo revelou para o grupo de pesquisadores reflexões acerca da importância de tratar o portador de lesão de forma holística, bem como de buscar alternativas de tratamento, através de estudos que investiguem as respostas às novas coberturas, melhorando a qualidade de vida dos pacientes acometidos por feridas crônicas através de métodos alternativos e de baixo custo, otimizando assim os recursos investidos nos setores de saúde. 


\section{REFERÊNCIAS}

BARBOSA, Maria Helena; ZUFFI, Fernanda Bonato; MARUXO, Harriet Bárbara; JORGE, Lívia Loamí Ruyz. Ação terapêutica da própolis em lesões cutâneas. Acta paul. enferm. São Paulo, v. 22, n. 3. 2009.

BEZERRA, Kévia Katiúcia Santos; BEZERRA, Anne Milane Formiga; SOUSA, Luci Cleide Farias Soares, SOUSA, José da Silva; SILVA, Rosilene Agra Da; BORGES, Maria da Gloria Borba. Atividade antimicrobiana da própolis em lesões cutâneas. ACSA. UFCG. v. 9 (1), 2013.

BORGES, Eline Lima; PIRES JÚNIOR, José Ferreira; ABREU, Mery Natali Silva; LIMA, Vera Lúcia de Araújo; SILVA, Patrícia Aparecida Barbosa; SOARES, Sônia Maria. Fatores associados à cicatrização de feridas cirúrgicas complexas mamária e abdominal: estudo de coorte retrospectivo. Rev. Latino-Am. Enfermagem, 24 (1). 2016.

GREGORY, Scott R., PICCOLO, Nelson; PICCOLO, Maria T.; PICCOLO, Monica S.; HEGGERS, John P.. Comparison of propolis skin cream to silver sulfadiazine: a naturopathic alternative to antibiotics in treatment of minor burns. J Altern Complement Med; 8(1). 2002.

OLIVEIRA, Simone Helena dos Santos; SOARES, Maria Julia Guimarães Oliveira; ROCHA, Pascalle de Sousa. Uso de cobertura com colágeno e aloe vera no tratamento de ferida isquêmica: estudo de caso. Rev. esc. enferm. USP, São Paulo, v. 44, n. 2. 2010.

SANTOS, Marinaldo José dos; VIANNA, Lucila do Amaral Carneiro; GAMBA, Mônica Antar. Avaliação da eficácia da pomada de própolis em portadores de feridas crônicas. Acta paul. enferm. São Paulo, v. 20, n. 2. 2007.

VARGAS, Agueda Castagna de; LOGUERCIO, Andrea Pinto; WITT, Niura Mazzini; COSTA, Mateus Matiuzzi da; SÁ E SILVA, Mariana; VIANA, Luciane Ribeiro. Atividade antimicrobiana "in vitro" de extrato alcóolico de própolis. Cienc. Rural, Santa Maria, v. 34 (1). 2004. 